\title{
Super Vasmol Poisoning: Dangers of Darker Shade
}

\author{
Subramanian Senthilkumaran ${ }^{1}$, Narendra N Jena ${ }^{2}$, Ponniah Thirumalaikolundusubramanian ${ }^{3}$ \\ Indian Journal of Critical Care Medicine (2019): 10.5005/jp-journals-10071-23303
}

\section{INTRODUCTION}

The ancient science of cosmetology is assumed to have originated in India and Egypt. Available evidences indicate that highly advanced ideas of hair care and hair dressing among men and women were practiced in ancient India. The earliest records of art of dyeing hair with henna, indigo, and vegetable dyes date back to circa 2500 and 1550 B.C., to the Indus valley civilization, and their preferred colors were black or orange-red. ${ }^{1}$ The secrets of art and nature as well as the various methods of hair coloring like black, gold, green, red, yellow, and white were described as early as $1661 .^{2}$ The development of synthetic dyes for hair in the laboratory is traced back to 1856 , and permanent hair dyes have been in commercial use more than 10 decades ago. ${ }^{3}$ The systemic toxicity of paraphenylenediamine (PPD) was first documented in a hairdresser handling dye in 1924 due to occupational exposure. Super Vasmol is an emulsion-based popular hair dye used in India, and the poisoning due to it is emerging as a major cause of suicide in the developing world since it is cheap and freely available overthe-counter. ${ }^{4}$

\section{Toxic Cocktail}

The active ingredients of Super Vasmol include PPD, propylene glycol, liquid paraffin, resorcinol, cetostearyl alcohol, sodium lauryl sulfate, sodium ethylenediaminetetraacetic acid, preservatives, and perfumes. ${ }^{5}$ The main compound responsible for the toxicity is paraphenyl-diamine $\left[\mathrm{C}_{6} \mathrm{H}_{4}\left(\mathrm{NH}_{2}\right)_{2}\right]$, which is a key ingredient for most of the hair dyes, and it is used for color enhancement. It is an aromatic amine derived from coal tar and is used for commercial purposes.

PPD is available in the form of white crystals when it is pure and rapidly turns to brown when exposed to air. It has a molecular weight of 108 Dalton with a boiling point of $267^{\circ} \mathrm{C}$ and melting point of $140^{\circ} \mathrm{C} .^{6}$ It is easily soluble in ethanol, ether, benzene, chloroform, and acetone and partially soluble with agitation in water. The concentration of PPD in hair dye formulation varies from $70 \%$ to $90 \%$ in stone hair dye and 2 to $10 \%$ in branded dyes.

A $100-\mathrm{mL}$ Super Vasmol contains $12 \mathrm{~g}$ of PPD, and the minimal dose required to produce systemic features of poisoning is approximately $3 \mathrm{~g}$. The fatal dose after oral ingestion is $7 \mathrm{~g}$ or more. ${ }^{7}$ Exact concentrations of PPD in different formulations are not known as these formulations are patented. Paraphenylenediamine is quickly absorbed into systemic circulation through mucous membranes of the digestive tract after oral intake. It is acetylated into $N$-acetyl-PPD and $\mathrm{N}, \mathrm{N}$-diacetyl-PPD as major metabolites for detoxification and excreted in the urine. Bandrowski's base is formed by the oxidation reaction of PPD with base in an alkaline medium, which is allergenic, mutagenic, and highly toxic. ${ }^{8}$
${ }^{1}$ Department of Emergency and Critical Care, Manian Medical Centre, Erode, Tamil Nadu, India

${ }^{2}$ Department of Emergency Medicine, Meenakshi Mission Hospital and Research Centre, Madurai, Tamil Nadu, India

${ }^{3}$ Department of Internal Medicine, Trichy SRM Medical College Hospital and Research Center, Irungalur, Tiruchirappalli, Tamil Nadu, India

Corresponding Author: Subramanian Senthilkumaran, Department of Emergency and Critical Care, Manian Medical Centre, Erode, Tamil Nadu, India, Phone: +91 9994634444, e-mail: maniansenthil@yahoo. co.in

How to cite this article: Senthilkumaran S, Jena NN, Thirumalaikolundusubramanian P. Super Vasmol Poisoning: Dangers of Darker Shade. Indian J Crit Care Med 2019;23(Suppl 4):S287-S289.

Source of support: Nil

Conflict of interest: None

Resorcinol found in hair dyes is a phenol derivative, which is chemically designated as 1,3-dihydroxybenzene or 3-hydroxyphenol. It is highly corrosive in nature. Propylene glycol is used as a solvent in the hair dyes. Among all the active ingredients of Super Vasmol PPD, resorcinol and propylene glycol are found to have systemic toxicity and has the most life-threatening effects. ${ }^{9}$

\section{Toxic Effects}

A three-stage progression has been witnessed in PPD poisoning with inflammatory stress characterized by a relative immunodepression in the first 3 days, proinflammatory state (due to rhabdomyolysis) from third to sixth day, and immunomodulative action (due to oxidative metabolism) from the sixth day.

It causes systemic inflammatory reaction specific to a cytotoxic cell effects. The pathophysiologic mechanisms could be due to increased free radical formation, skeletal and cardiac muscle necrosis (scattered coagulation necrosis), formation of highly nephrotoxic quinone diamine (an oxidation product of PPD metabolites), renal tubular occlusion due to myoglobin casts, and acute tubular necrosis. Hoarseness of voice, pneumothorax, ${ }^{10}$ hepatitis, ${ }^{11}$ convulsions, coma, cardiac toxicity, hypotension, and sudden cardiac death are on the toxic end of the spectrum.

\section{Clinical Features}

The characteristic triad of Super Vasmol poisoning is early angioneurotic edema of face and neck with hoarseness of voice and stridor, rhabdomyolysis with dark brown colored urine and acute kidney injury (AKI) even in the absence of laboratory facilities or in the absence of proper history. ${ }^{12}$ These patients have obvious orofacial swelling with swollen hard protruding tongue and

(c) The Author(s). 2019 Open Access This article is distributed under the terms of the Creative Commons Attribution 4.0 International License (https://creativecommons. org/licenses/by-nc/4.0/), which permits unrestricted use, distribution, and non-commercial reproduction in any medium, provided you give appropriate credit to the original author(s) and the source, provide a link to the Creative Commons license, and indicate if changes were made. The Creative Commons Public Domain Dedication waiver (http://creativecommons.org/publicdomain/zero/1.0/) applies to the data made available in this article, unless otherwise stated. 
edematous bull neck, which are the earliest manifestation following ingestion of the dye.

The most bulbous edema is provoked by PPD, and it appears to be exceptionally specific and selectively limited in the head and neck. It was suggested that the toxic effect of the PPD is due to its oxidation product quinone diamine, which is responsible for severe local irritation. Some authors thought that PPD toxicity is due to the consequence either on the blood colloids or on vascular permeability. Moreover, it was understood that the PPD toxicity is due to altered vascular permeability and involvement of the parasympathetic nervous system. ${ }^{13}$ Deamination and formation of aniline from PPD are also claimed to be accountable in part for the toxic symptoms. The differences in opinion as to the reason of PPD edema appear due to the fact that the exact number and nature of the oxidation products are not known. These results are dependable based on the view that oxidative stress may be an essential part of the preimmunological phase in the induction of the allergic contact dermatitis by PPD.

Super Vasmol is a potent nephrotoxic cocktail; the extent of renal involvement in hair dye poisoning varies between transient proteinuria and oliguric AKI. Acute kidney injury is a late manifestation of PPD poisoning, which usually develops few days after PPD exposure. The development of AKI in PPD intoxication does not occur in all patients, and it differs from $47.3 \%$ to $100 \%$. Naqvi et al. had reported a cohort of hundred patients presented to his institution with AKI following PPD poisoning. ${ }^{14}$

The mechanisms of AKI following hair dye poisoning are several. Paraphenylenediamine itself has a direct toxic effect on kidney due to its aromatic structure, making its reabsorption and concentration in tubule easy, and it can also cause acute tubular necrosis. Rhabdomyolysis is the main cause of renal failure and is a recognized complication of hair dye poisoning. ${ }^{15}$ Paraphenylenediamine causes rhabdomyolysis by promoting calcium release and leakage of calcium ions from the smooth endoplasmic reticulum, followed by continuous contraction and irreversible change in the muscle's structure, which results in deposition of myoglobin cast within the renal tubules, and hemolysis leading to acute tubular necrosis and AKI. ${ }^{16}$ Hypovolemia and toxic metabolites of PPD also contribute to AKI. Propylene glycol is associated with hyperosmolality, raised anion gap metabolic acidosis, central nervous system depression, arrhythmias, and renal dysfunction.

The respiratory distress following the oral ingestion of Super Vasmol leads to severe airway compromise as a result of direct trauma to the tissues by chemicals and causes dyspnea and asphyxia secondary to inflammatory edema involving upper airway which often warrants an emergency airway intervention. ${ }^{17}$ Suliman et al. ${ }^{18}$ noted angioneurotic edema in $68 \%$ and emergency tracheostomy in $15.8 \%$. Senthilkumaran et al. ${ }^{19}$ had reported alveolar rupture in two cases probably secondary to the trapping of large volume of air due to intense inspiratory effort as a result of laryngeal edema, which might have contributed to over-distension of the alveoli and interstitial pulmonary air leak, rather than rupture of bullae. In an autopsy series of eight cases of hair dye poisoning, pneumothorax was noticed in two without any other demonstrable pathology. ${ }^{20}$ In view of clinical observations and autopsy evidences of pneumothorax in hair dye poisoning, physicians treating such cases have to consider the possibility of pneumothorax if the patient has respiratory distress, as timely intervention saves lives. Myocarditis is a fatal and a frequently ignored complication of
PPD poisoning, due to lack of awareness of this complication among clinician and scarcity of data in the medical literature. The incidence of myocarditis was $15 \%$, with a high mortality rate of $29 \% .^{21}$ However, the occurrence is bound to vary, with patients differing in their vulnerability to PPD, apart from the amount of dye ingested. Exposure to hair dye chemicals may lead to intravascular hemolysis, hemoglobinuria and methemoglobinemia. ${ }^{22}$

\section{Treatment}

The management of Super Vasmol poisoning is mainly dependent on maintaining airway patency and preventing acute renal failure. Currently, no specific antidote is available to neutralize the toxic effects of PPD. Hence, the treatment is mainly supportive and depends on the clinical situation. It is preferable to consider tracheostomy over cricothyroidotomy as an emergency surgical airway in the presence of extensive neck swelling and difficulties encountered in identifying landmarks. However, endotracheal intubation done in early stages of edema or prophylactic intubation before development of edema can avoid tracheostomy. Antihistamines and steroids are commonly used in the management of airway edema possibly due to hypersensitivity reaction to PPD even though there is no therapeutic trial to substantiate their benefits.

Timely institution of alkaline diuresis using isotonic saline, sodium bicarbonate, and osmotic diuretics and maintenance of a high urine output can prevent acute renal failure due to rhabdomyolysis. Paraphenylenediamine is non-dialyzable compound and dialysis is only part of the supportive therapy. However, trials of PPD removal were attempted using hemoperfusion and hemodialysis, with variable results. The most consistent predictors of mortality are the amount of hair dye ingested, the time interval between consumption and intervention, hyperkalemia, hypocalcemia, and hyperphosphatemia; and mortality rates varied between $0.03 \%$ and $60 \%$.

Interestingly PPD is banned in certain countries like Germany, France, Sweden, Sudan, etc. Free availability contributes to Super Vasmol poisoning, but restricted use or introduction of alternatives may likely bring down the incidence of Super Vasmol poisoning. In addition, community awareness and health education help towards early diagnosis and prevention to some extent.

\section{Learning Points}

- A diagnosis of Super Vasmol poisoning is made invariably on the basis of characteristic clinical trial.

- Early endotracheal intubation can preclude tracheostomy.

- Possibility of pneumothorax has to be considered if hypoxia persists or worsens or in case of cardiorespiratory arrest.

- Serum creatinine kinase level is not a reliable indicator of myocardial injury.

- Urine may not contain myoglobin if the patient presents late.

\section{References}

1. Wolfram LJ. Hair cosmetics. In: Barel AO, Paye M, Maibach HI, ed. Handbook of Cosmetic Science and Technology. New York, NY, USA: Marcel Dekker; 2001.

2. Sherrow V. Encyclopedia of Hair: A Cultural History. Westport, CT, USA: Greenwood Press; 2006. 
3. Harrison S, Sinclair R. Hair colouring, permanent styling and hair structure. J Cosmet Dermatol 2003;2(3-4):180-185. DOI: 10.1111/j.1473-2130.2004.00064.x.

4. Sampathkumar K, Yesudas S. Hair dye poisoning and the developing world. J Emerg Trauma Shock 2009;2(2):129-131. DOI: 10.4103/09742700.50749.

5. Kumar PA, Talari K, Dutta TK. Super vasomol hair dye poisoning. Toxicol Int 2012;19(1):77-78. DOI: 10.4103/0971-6580.94503.

6. Scientific Committee on Consumer Safety (SCCS) opinion on p-Phenylenediamine. COLIPA no. A7. SCCS/1443/11. Revision of 18 September 2012. http://ec.europa.eu/health/scientific_committees/ consumer_safety/index_en.htm. Accessed on November 17, 2019.

7. GudeD, BansalDP,Ambegaonkar R, Prajapati J.Paraphenylenediamine: Blackening more than just hair. J Res Med Sci 2012;17(6):584-586.

8. Gibson A, Kim SH, Faulkner L, Evely J, Pirmohamed M, Park KB. In vitro priming of Naïve T-cells with p-phenylenediamine and Bandrowski's base. Chem Res Toxicol 2015;28(10):2069-2077. DOI: 10.1021/acs. chemrestox.5b00294.

9. Chrispal A, Begum A, Ramya I, Zachariah A. Hair dye poisoning - an emerging problem in the tropics: an experience from a tertiary care hospital in South India. Trop Doct 2010;40(2):100-103. DOI: 10.1258/ td.2010.090367.

10. Senthilkumaran S, Thirumalaikolundusubramanian P. Acute hair dye poisoning: Lurking dangers. J Mahatma Gandhi Inst Med Sci 2015;20:33-37. DOI: 10.4103/0971-9903.151731.

11. Ishtiaq R, Shafiq S, Imran A, Masroor Ali Q, Khan R, Tariq H, et al. Frequency of acute hepatitis following acute paraphenylene diamine intoxication. Cureus 2017;9(4):e1186. DOI: 10.7759/cureus.1186.

12. Garg SK, Tiwari R, Ahlawat A. Hair dye poisoning: an unusual encounter. Indian J Crit Care Med 2014;18(6):402-404. DOI: 10.4103/0972-5229.133941.

13. Tainter ML, Hanzlik PJ. The mechanism of edema production by paraphenylene diamine. J Pharmacol Exp Ther 1924;24:179-211.
14. Naqvi R, Akhtar F, Farooq U, Ashraf S, Rizvi SA. From diamonds to black stone; myth to reality: acute kidney injury with paraphenylene diamine poisoning. Nephrology (Carlton) 2015;20(12):887-891. DOI: 10.1111/nep.12534.

15. Sampathkumar K, Sooraj YS, Ajeshkumar RP, Mahaldar AR, Muthiah R. Rhabdomyolysis due to hair dye poisoning: an emerging threat. Indian J Crit Care Med 2007;11:212-214. DOI: 10.4103/0972-5229. 37717.

16. Bokutz M, Nasir N, Mahmood F, Sajid S. Hair dye poisoning and rhabdomyolysis. J Pak Med Assoc 2015;65(4):425-426.

17. Umair SF, Amin I, Urrehman A. Hair dye poisoning: "An early intervention". Pak J Med Sci 2018;34(1):230-232. DOI: 10.12669/ pjms.341.14123.

18. Suliman SM, Fadlalla M, Nasr Mel M, Beliela MH, Fesseha S, Babiker $M$, et al. Poisoning with hair-dye containing paraphenylene diamine: ten years experience. Saudi J Kidney Dis Transpl 1995;6(3): 286-289.

19. Senthilkumaran S, Ram J, Menezes RG, Sweni S, Thirumalaikolundusubramanian P. Pneumothorax in hair dye poisoning: an unrecognized danger. Lung India 2011;28(4):323-324. DOI: 10.4103/0970-2113.85753.

20. Senthilkumaran S, Suresh P, Menezes RG, Thirumalaikolundusubramanian P. Fatal hair dye poisoning-autopsy study. Forensic Int 2009;12:23-25.

21. Balasubramanian $D$, Subramanian $S$, Thangaraju $P$, Shanmugam $K$. Right bundle branch block: an uncommon cardiotoxic manifestation of hair dye poisoning-a case report. J Clin Diagn Res 2014;8(1):174-176. DOI: 10.7860/JCDR/2014/6966.3962.

22. Senthilkumaran S, David SS, Menezes RG, Thirumalaikolundusubramanian P. Henna leaf ingestion and intravascular hemolysis: the missing link. Saudi J Kidney Dis Transpl 2014;25(3): 667-668. DOI: 10.4103/1319-2442.132232. 